\title{
Shape Characterization of the Corpus Callosum in Schizophrenia Using Template Deformation
}

\author{
Abraham Dubb, Brian Avants, Ruben Gur, and James Gee \\ Departments of Bioengineering, Psychiatry and Radiology \\ University of Pennsylvania \\ Philadelphia, PA, USA 19104 \\ \{adubb, avants\}@grasp.cis.upenn.edu \\ gur@bblmail.psycha. upenn.edu \\ gee@rad.upenn.edu
}

\begin{abstract}
The presence of morphologic differences in the corpus callosum in people with schizophrenia has been the subject of intense investigation for a number of years. Researchers, however, have been unable to produce consistent results through comparison of total and partitioned areas. As an alternative to these indices, we use template deformations to find regional size variations in a population of 100 patients. We generated a set of Jacobian determinant maps using $k$-cluster segmentation followed by a curve registration algorithm. We performed several comparisons including control versus schizophrenia, control versus schizophrenia within gender and age-related effects of control versus schizophrenia. Statistical plots revealed a substantial area of contraction in the anterior callosum associated with schizophrenia. Gender stratification showed a large contribution of this effect was from females. In addition, patients failed to demonstrate an age-related expansion of the splenium that was present among controls. Our results show that template deformation morphometry can be used to show morphologic differences in the callosa of people with schizophrenia.
\end{abstract}

\section{Introduction}

The corpus callosum is the largest white-matter tract in the human brain and serves as the primary means of communication between the two cerebral hemispheres. In many instances, higher cortical function is mediated by this tract as it allows integration of cortical processes from opposite sides of the brain. The corpus callosum has attracted much attention from researchers in several fields including neurology, psychiatry and computer science. There are several reasons for this. First, the corpus callosum is easily identified on a mid-sagittal section of the brain. Its white matter fibers in cross-section stand out in stark relief against the surrounding cingulate gyrus and lateral ventricles. This anatomical property also aids in efficient automated segmentation of the corpus callosum. Second, the actual shape of the callosum is fairly well preserved with only subtle phenotypic variations present in the general population. This factor aids in 
template matching and template deformation morphometry (TDM). Finally, the literature provides evidence that the corpus callosum may be involved in a number of disorders including schizophrenia, Alzheimer's dementia [1] and mental retardation [2]. Many have attempted to relate the actual cross-sectional shape of this tract to such conditions.

The role of the corpus callosum in schizophrenia has been the subject of much interest for a number of years. Rosenthal [3] peformed a post-mortem comparison of brains of people with schizophrenia versus control and showed a $1 \mathrm{~mm}$ increase in thickness in the callosa of patients. The advent of MRI has allowed researchers to study callosa in vivo in order to discover morphologic differences. Traditionally, researchers have used length, thickness and cross-sectional area as indices to compare with controls. A major difficulty with this approach lies in whether or not to normalize the callosa for brain size. Normalization of the corpus callosum is confounded by the fact that the relationship between callosal size and brain volume is not linear, and brain volume, itself, is decreased in schizophrenia 4. Even if one attempts to normalize the callosum, debate exists on how best to do this. For example, do we normalize to brain volume, total mid-sagittal area or to the anterior-posterior (AP) dimension of the brain? The difficulty of using area as an index is well demonstrated in a meta-analysis of callosa size in schizophrenia performed by Woodruff [4]. Of the 11 studies that were included in the final analysis, 8 demonstrated greater callosal area in controls while the other 3 yielded the opposite result. Perhaps more importantly, only 3 of the 11 were able to achieve statistical significance.

A more recent method for studying the callosum involves dividing the crosssection of the tract into partitions and comparing regional area instead of total area. Downhill [5] used this technique in a comparison of callosa from 27 people with schizophrenia and 30 controls. In this study, the callosum was divided into $30 \mathrm{AP}$ sectors and statistical analysis showed that the genu and splenium to be smaller in schizophrenia. While this approach may seem like a viable alternative to total area, the normalization question is not resolved. Furthermore, selection of a partitioning method is arbitrary since no one has estabilished the best way to divide up the callosum.

Given the issues raised with area calculation and the historically inconclusive results associated with this technique, we chose to apply a registration and TDM approach in order to demonstrate local size variations within the callosum 6] [8]. Pettey and Gee [8] describe this approach and apply it to the comparison of male and female callosa. Our current study makes use of a curve registration algorithm over the extracted callosal boundaries, while Pettey directly registered images of the callosa. The underlying premise, however, is identical. TDM offers several advantages over more traditional methods of callosal analysis; TDM provides information on the regional shape of a structure rather than simply overall size, offering a potentially richer and more flexible description of anatomic variations. Furthermore, we believe that TDM can be used to demonstrate phenotypic variation for a wide array of neuroanatomical structures. 


\section{Methods}

\subsection{Subjects and Data Acquisition}

The Schizophrenia Center at the University of Pennsylvania maintains a database containing hundreds of cranial MRI's of both psychiatric patients and healthy volunteers. Our selection criteria specified right handed participants with a diagnosis of schizophrenia or control, for whom a $1 \mathrm{~mm}$ slice thickness scan was available. While there were many MRI's available using $5 \mathrm{~mm}$ slice thickness, we opted to exclude these studies on the basis that their inferior pixel resolution would make them poor substrates for TDM analysis. Magnetic resonance imaging scans were acquired on a $1.5 \mathrm{~T}$ scanner (Signa; General Electric Co., Milwaukee, WI) with a spoiled gradient recalled pulse sequence using the following parameters: flip angle of $35^{\circ}$, repetition time of 35 milliseconds, echo time of 6 milliseconds, field of view of $24 \mathrm{~cm}, 1$ repetition, $1 \mathrm{~mm}$ slice thickness and no interslice gaps. Transaxial images were in planes parallel to the orbitomeatal line, with resolution of $0.9375 \times 0.9375 \mathrm{~mm}^{2}$.

\subsection{Reslicing}

Our goal in reslicing the brain volumes was to obtain the most precise midsagittal image possible. This procedure was necessitated by the fact that most participants had tilted their heads slightly to the left or right side during the scan. We adopted the reslicing protocol used by the Schizophrenia Center by performing the following steps: first, the volume was rotated around a manually drawn anterior-posterior axis until the eyeballs were positioned in the same horizontal plane. Next, an approximate mid-sagittal image was obtained by manually drawing the slice plane through mid-line structures at the level of the interventricular septum. Third, the sagittal plane was moved laterally until the falx cerebri and uninterrupted central canal (at the level of the midbrain) were present in the same plane. In some cases, the volume was tilted slightly around the AP axis until both structures were present in a single plane. This mid-sagittal image was then extracted and rotated around its center so that the anterior-commissure and posterior-commissure were horizontal. Figure 1 shows one sample mid-sagittal image processed in this way.

\subsection{Segmentation}

We employed a $k$-means clustering algorithm which groups image voxels into $k$ clusters by minimizing the intensity variance within each cluster [10]. Because the performance of this algorithm was highly dependent on the brightness of the input image and number of clusters, we opted to perform cluster segmentation on the same data-set using $k=3,4$, and 5 and scaled at multiple brightness levels. This process yielded 18 sets of segmentation images $(3$ segmentation levels $\times 6$ brightness settings). For each mid-sagittal image, the best segmentation image from the set of 18 was chosen and modified so that the callosa was completely 

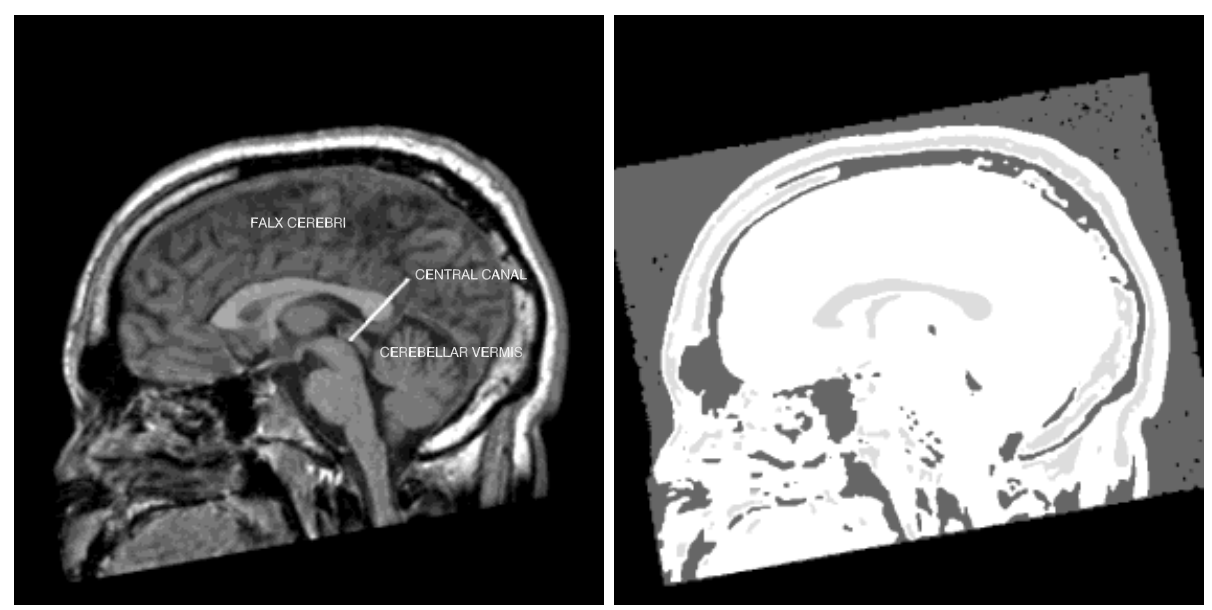

Fig. 1. Raw mid-sagittal image (left) and segmentation mask (right). The raw image on the left shows the three anatomical structures which signify a mid-sagittal slice. The frequent presence of the interventricular septum within this slice complicates segmentation. On the right, a successful $k$-means probability based segmentation image which shows the corpus callosum as a solitary island.

separated from surrounding structures. The modified image was then used to extract the corpus callosum from the mid-sagittal section. Figure 1 shows one sample segmentation image.

Once we have generated the set of segmented callosa, our next step was to crop the images and perform registration and deformation to a single template callosum. While selection of a particular registration method probably has a profound impact on the final results, choosing the optimal algorithm remains a subject of debate. Our lab is currently involved in an ongoing study to determine the effect that registration method and template selection may have on final results. In this particular case, we applied a curve-registration algorithm using a male control as a template.

\subsection{Callosal Matching}

We want to find a vector field that smoothly maps the image $I_{1}$ to image $I_{2}$, such that the borders and interiors of the anatomy are well matched. We base this mapping entirely on the boundary of the callosa for two reasons. First, for our purposes, the callosa is completely defined by the shape of its boundary. Second, a globally optimal solution to the one dimensional correspondence problem can be found with dynamic programming [7]. We then interpolate the boundary correspondence to the interior of the shapes.

Our formulation of the matching problem [9] is in terms of the variational calculus. We want to find a monotonic path through the $s_{1} \times s_{2}$ reparameterization space,where $s$ denotes the arc length parameter, so that the template callosal 
curve is better matched with a subject curve. Solutions to Laplace's equation are well known to be maximally smooth. We thus choose to find $g: s_{1} \rightarrow s_{2}$ that minimizes the integral form of Laplace's equation. This gives the energy functional,

$$
E(g)=\frac{1}{2} \int_{0}^{1}\left\|\nabla V\left(s_{1}\right)\right\|^{2} d s_{1}
$$

where

$$
V\left(s_{1}\right)=C_{2}\left(g\left(s_{2}\right)\right)-C_{1}\left(s_{1}\right)
$$

We note that this formulation is inherently asymmetric, but that the asymmetry has little consequence in this application. We then use this solution as a boundary condition for solving Laplace's equation on the reference callosum interior. Discretization and successive over relaxation allows the Euler-Lagrange form of the following energy function to be minimized efficiently,

$$
E(F)=\frac{1}{2} \int_{s_{1}}(F-V)^{2} d s_{1}+\frac{1}{2} \int_{\Omega}\|\nabla F\|^{2} d \Omega .
$$

Here, $F$ is the vector field on the interior, $\Omega$. Note that we minimize the same energy for the boundary and interior.

\subsection{Statistical Analysis of the Jacobian}

The registration map $F$ may be viewed as a deformation field that takes the pixels of the template callosa to those of the subject callosa. The deformation field is defined as a set of $N$ displacement vectors. The following equation

$$
\mathbf{u}_{k}=\left(u_{k}^{1}, u_{k}^{2}\right)
$$

gives the displacement vector needed to bring the $k^{t h}$ pixel of the template to the corresponding pixel of the patient. Using the vector field, $\mathbf{u}_{s}$, which describes the displacement field that relates the atlas to subject callosum, $s$, we can generate the following transformation equation:

$$
T_{s}(\mathbf{x})=\mathbf{x}+\mathbf{u}_{s}(\mathbf{x})
$$

$T_{s}(\mathbf{x})$ gives the corresponding position in subject callosum $s$ for pixel $\mathbf{x}$ in the template. In order to describe the expansion or contraction that occurs at each pixel in this transformation, we use the following quantity:

$$
\left|\frac{\partial T_{s}}{\partial \mathbf{x}}\right|
$$

which is known as the determinant of the Jacobian of the transformation. For ease of notation, we will simply refer to this value as the Jacobian or $J$. In order to normalize the Jacobian for global variation [8], we scale this quantity at each pixel, $k$ :

$$
j_{k}=\frac{J_{k}}{\sum_{k} J_{k}} .
$$


If we are comparing the Jacobians of controls with patients, we may calculate the t-score at each pixel, $k$ :

$$
t_{k}^{+}=\frac{\overline{j_{k}^{c}}-\overline{j_{k}^{s}}}{\sigma_{k} \sqrt{\frac{1}{N_{s}}+\frac{1}{N_{c}}}}, \quad t_{k}^{-}=\frac{\overline{j_{k}^{s}}-\overline{j_{k}^{c}}}{\sigma_{k} \sqrt{\frac{1}{N_{s}}+\frac{1}{N_{c}}}}
$$

where

$$
\sigma_{k}=\sqrt{\frac{\left(N_{c}-1\right) \sigma_{c}^{2}+\left(N_{s}-1\right) \sigma_{s}^{2}}{N_{c}+N_{s}-2}} .
$$

$\overline{j_{k}^{c}}$ and $\overline{j_{k}^{s}}$ are the mean normalized Jacobians for the control and schizophrenia groups at the $k^{t h}$ pixel, respectively. $\sigma_{c}$ and $\sigma_{s}$ are the standard deviations of $j_{k}$ for controls and patients, respectively. $N_{c}$ and $N_{s}$ are the number of subjects in the control group and schizophrenia group. We calculate the t-score in both directions so that we can differentiate areas of contraction and expansion. The t-scores, $t_{k}^{+}$and $t_{k}^{-}$, may now be converted to p-values using the Student's t cumulative distribution function and then threshold plotted over the template mask. We performed these series of calculations using several different subpopulations, including control/schizophrenia, control/schizophrenia within sex, and control/schizophrenia within age groups.

\section{Results}

Our query of the Schizophrenia Center database yielded a total of 290 cranial MRI's, 190 control and 100 schizophrenic. The average age among these subgroups was $28.0 \pm 9.9$ years and $33.7 \pm 11.2$ years, respectively. A number of findings are present in the statistical plots shown in figure 2. Figure 2a shows the comparison between controls and schizophrenia. There is a substantial area of contraction in the anterior third of callosa in patients as compared to controls. There is a small relatively insignificant area of expansion in the mid-body. Stratification between the sexes show that the anterior callosa are more contracted in female than in male patients. The small area of mid-body expansion seems to be due solely to an effect in males. In contrast, females with schizophrenia have a focal area of expansion in the caudal splenium that does not exist in males.

The comparison of age-related effects reveals a pronounced difference. Healthy subjects aged 36 to 55 were found to have a larger splenium than their younger counterparts. This splenial expansion is virtually absent in the corresponding age-related study of patients. Moreover, there is a relatively insignificant area of expansion within the isthmus among older patients that is not present in older controls.

\section{Discussion}

The goal of this paper was to demonstrate the utility of template deformation morphometry in showing subtle morphologic differences in the corpus callosum 


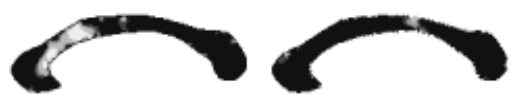

(a) Controls vs. patients

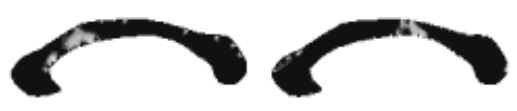

(b) Male controls vs. male patients

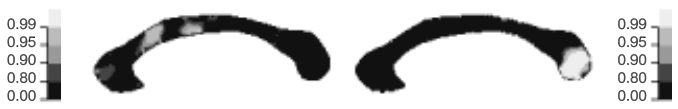

(d) Young controls vs. old controls
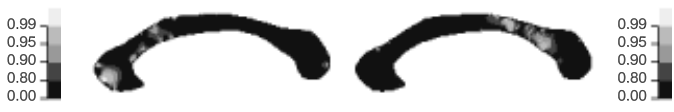

(e) Young patients vs. old patients

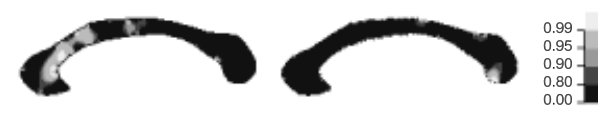

(c) Female controls vs. female patients

Fig. 2. P-value plots for five different subpopulation comparisons. Figures on the left of each panel show areas of contraction in the study group in comparison to controls, whereas figures on the right show areas of expansion in the study group. Figure 2a compares controls with patients. Figure $2 \mathrm{~b}$ compares male controls with male patients. Figure 2r compares female controls with female patients. The last two sets of callosa compare age-related effects in controls and then in patients. The left side shows areas of contraction in the older population. Figure $2 \mathrm{~d}$ compares controls aged 18-35 with controls aged 36-55. Figure 22 compares patients aged 18-35 with patients aged 36-55.

of patients versus controls. Using this method, we discovered localized regions where the shape of the callosa was different in patients. It is noteworthy that our result showing contraction of the anterior third of the callosa in patients is opposite from the findings of Narr 11. Using a parametric surface modeling approach, Narr et. al. showed that the anterior callosal segment was thicker in patients with schizophrenia. Narr's study was limited, however, by a relatively small study population (25 patients and 28 controls).

The comparison of age-related changes merits a closer look. Bartzokis [12] studied the age-related changes in the frontal and temporal lobes in men. He found that while gray matter decreases with age, men undergo an age-related expansion of white matter up to age 47. In a more recent study, Bartzokis [13] found that this normal age-related white matter expansion was absent in patients with schizophrenia. These findings are consistent with our results showing a relatively decreased degree of splenial expansion in older patients.

The question of morphologic changes in the callosum of people with schizophrenia is far from resolved. Methods of analysis continue to dictate results. Through the application of template deformation morphometry, we hope we can produce results that are more objective and robust than those of more traditional methods. 


\section{Acknowledgements}

This work was supported in part by the USPHS under grants NS-33662, LM03504, MH-62100, AG-15116, AG-17586 and DA-14418.

\section{References}

1. Pantel, J., Schroder, J., Jauss, M., Essig, M., Minakaran, R., Schonknect, P., Schneider, G., Schad, L. R., Knopp, M. V.: Topography of Callosal Atrophy Reflects Distribution of Regional Cerebral Volume Reduction in Alzheimer's Disease. Psychiatry Research. 90 (1999) 180-192

2. Marszal, E., Jamroz, E., Pilch, J., Kluczewska, E., Jablecka-Deja, H., Krawczyk, R.: Agenesis of Corpus Callosum: Clinical Description and Etiology. J. of Child Neurology. 15 (2000) 401-405

3. Rosenthal, R., Bigelow, L. B.: Quantitative Brain Measurements in Chronic Schizophrenia. Br. J. of Psychiatry. 121 (1972) 259-64

4. Woodruff, P. W. R., McManus, I. C., David A. S.: Meta-Analaysis of Corpus Callosum Size in Schizophrenia. Neurology, Neurosurgery, \& Psychiatry. 58 (1995) 457-461

5. Downhill Jr., J. E., Buchsbaum, M. S., Wei, T., Spiegel-Cohen, J., Hazlett, E. A., Haznedar, M. M., Silverman, J., Siever, L. J.: Shape and Size of the Corpus Callosum in Schizophrenia and Schizotypal Personality Disorder. Schizophrenia Research. 42 (2000) 193-208

6. Davatzikos, C., Resnick, S.: Sex Differences in Anatomic Measures of Interhemispheric Connectivity: Correlations with Cognition in Women but not Men. Cerebral Cortex. 8 (1998) 635-640

7. Amini, A., Weymouth, T., Jain, R.: Using Dynamic Programming for Solving Variational Problems in Vision. IEEE Transactions on Pattern Analysis and Machine Intelligence. 12 (1990) 855-867

8. Pettey, D. J., Gee. J. C.: Sexual Dimorphism in the Corpus Callosum: a Characterization of Local Size Variations and a Classification Driven Approach to Morphometry. (2002) submitted

9. Avants, B., Gee, J. C.: Soft Parametric Curve Matching in Scale-Space. SPIE Medical Imaging. (2002) in press

10. Machado, A. M. C., Gee, J. C.: Atlas Warping for Brain Morphometry. SPIE Medical Imaging. (1998) 642-651

11. Narr, K. L., Thompson, P. M., Sharma, T.: Mapping Morphology of the Corpus Callosum in Schizophrenia. Cereberal Cortex. 10 (2000) 40-49

12. Bartzokis, G., Beckson, M., Lu, P. H., Nuechterlein, K. H., Edwards, N., Mintz, J.: Age-Related Changes in Frontal and Temporal Lobe Volumes in Men: A Magnetic Resonance Imaging Study. Arch. of General Psychiatry. 58 (2001) 461-465

13. Bartzokis G., Nuechterlein, K. H., Lu, P. H., Mintz, J.: Dysregulated Brain Development in Adult Men with Schizophrenia: a Magnetic Resonance Imaging Study. Arch. of General Psychiatry. (2001) submitted 